\title{
Pensamiento crítico en los alumnos de la Universidad Nacional de Trujillo- 2014
}

\section{Critical thinking in the student of National Trujillo University -2014}

\author{
Tamara Pando ${ }^{1}$
}

\section{RESUMEN}

La presente investigación se realizó con el propósito de determinar el desarrollo del pensamiento crítico a través de un programa de lectura comprensiva en los alumnos de la Universidad Nacional de Trujillo 2014

El estudio fue de tipo cuasi-experimental dentro de lo cual se tuvo el diseño pretest y postest con dos grupos, con una muestra de 72 alumnos, 30 hombres y 42 mujeres. Se procedió en primer lugar a la evaluación de los alumnos con el pretest en forma individual y con tiempo de 1 hora. A partir de entonces se procedió a aplicar el programa de lectura comprensiva de textos. Al finalizar la aplicación del programa se procedió a la aplicación del post-test. Se hicieron uso de los métodos cuantitativos, que se emplearon antes y después de la experiencia; y el método explicativo, para investigar acerca de las causas del porqué nuestro estudiante universitario no critica en los diversos espacios sociales del mundo que lo rodea.

Los resultados obtenidos muestran que luego de aplicar el post-test al grupo experimental y control, se incrementaron significativamente los porcentajes en los niveles, literal, inferencial y crítico; por lo tanto existe una relación estadística positiva. Se concluye que la aplicación del programa de lectura comprensiva de textos desarrolla significativamente el pensamiento crítico en los alumnos, la cual debe ser reforzada desde el nivel educativo, con la finalidad de lograr competencias laborales y asegurar la empleabilidad de los estudiantes.

Palabras claves: Programa de lectura comprensiva de textos, pensamiento crítico.

\section{ABSTRACT}

This research was conducted in order to determine the development of critical thinking though a comprehensive reading program in the students of the National Trujillo University 2014.

The study was quasi-experimental. The design was pre-test and post-test the experiment was divided into two groups. The sample was composed of 72 students; there were 30 men and 42 women. First the students were evaluated with an individual pre-test, which last one hour, and then we applied a comprehensive reading program of texts. At the end of the program we proceeded to apply of the post-test. The study has used quantitative methods; the same were used before and after the experience and we also used the explanatory method to investigate the causes of why our college students do not criticize in the various social problems surrounding the world.

The results show that after applying the post-test to the experimental group and the control one the percentages of the level were significantly increased, literal inferential and critical. Therefore there is a positive statistical relationship. To conclude the application of a comprehensive reading program of texts

1. Mg. Universidad Nacional de Trujillo. Dra. en Ciencias de la Educación, Universidad Nacional Mayor de San Marcos email: tamara-tatiana2010@hotmail.com 
develops significantly the critical thinking in students. This need to be reinforced from educational level, with the purpose of achieving job skills and ensure the students employability

Key words: comprehensive reading program of texts, critical thinking

\section{INTRODUCCIÓN}

Dentro del enfoque cognitivo se concibe la lectura tanto como proceso y como producto, de la interacción entre el texto y el lector. En el proceso de reconstruir las ideas del autor influyen muchos factores, siendo los más importantes, según Johnston (citado por Mendrano, 2012:6), “el texto, los conocimientos previos del lector y la relación autor lector". Cada uno de éstos es crucial a la hora de estimar el desarrollo del pensamiento crítico. Pensamiento crítico no es pensar por pensar, pero sí es el pensamiento que comporta el auto-mejoramiento.

Richard, P. (2000) sostiene que "este mejoramiento proviene de la habilidad para usar estándares por medio de los cuales uno evalúa apropiadamente el propio pensamiento. En otras palabras, es auto-mejoramiento (al pensar) a través de estándares (que evalúan el pensamiento)" (p.107).

Por el contrario, los pensadores críticos en el sentido "fuerte" del término, se someten a los mismos patrones intelectuales que sus oponentes, se atreven a reconocer la verdad en los puntos de vista opuestos, tienen habilidad para pensar multilógica y dialógicamente, son capaces de detectar sus pensamientos autodelusivos y se comprometen a vivir conforme a los principios críticos de su propio pensamiento.

Dewey, J. (1967) en su artículo "La concepción democrática en educación", considera que "el pensamiento y conocimiento son dos categorías interdependientes. Por una parte el pensamiento es esencial para la adquisición del conocimiento $y$, por la otra, el conocimiento es esencial para desarrollar el pensamiento." (p. 48).
Dewey pensaba que "el conocimiento práctico se puede asimilar eficazmente sí el estudiante lo procesa activamente por una vía intelectiva. Sólo luchando de cerca con las condiciones del problema, buscando y hallando sus propias salidas, se llega a pensar. Asimismo, existe la evidencia de la dependencia del pensamiento respecto del conocimiento. Podemos incluso definir un pensamiento hábil como la capacidad de aplicar el conocimiento de un modo eficaz" (p. 70). Un aspecto importante de la educación consiste en enseñar a la gente a pensar $y$, a pensar eficazmente: en síntesis, pensar de modo crítico.

Botkin, J. (1980) sostiene al respecto que el "Pensamiento es experimental y social hace una distinción entre el aprendizaje de mantenimiento y el aprendizaje innovativo" (p. 175).

El primero hace referencia a adquirir metodos y reglas que le servirán para hacer frente a situaciones conocidas, lo cual aumenta la capacidad que tiene la persona para resolver problemas, por ello se adecúa a un modo de vida ya establecido pero sin cambiarlo.

\section{¿Por qué es importante el estudio del pensamiento} crítico? En estudiantes universitarios, es una pregunta que puede ser abordada desde perspectivas diferentes, separadas por razones metodológicas pero relacionadas entre ellas, vínculos que se van haciendo evidentes en la medida en que cada una es expuesta.

Desde un punto de vista puramente etimológico, se revela su importancia a medida que se desvelan las raíces griegas y latinas de las cuales se derivan palabras como "crítico" y "pensamiento". 
A nivel social, la formación del pensamiento crítico se revela importante teniendo en cuenta el papel fundamental que desempeña en procesos sociales de naturaleza democrática en los cuales sea posible la formación de ciudadanos y ciudadanas, conscientes y responsables. Desde una perspectiva pedagógica, este tema y su importancia son avalados por instituciones como la Organización de las Naciones Unidas para la Educación, la Ciencia y la Cultura (UNESCO), que formula principios orientadores para la educación universitaria, en los cuales el desarrollo del pensamiento crítico ocupa un papel fundamental (Marciales, 2003:12).

Teniendo en cuenta lo anterior, pensar críticamente cobra importancia fundamental en un mundo que, agobiado por las crisis en todos los órdenes, sociales, políticos, y económicos entre otros, demanda cada vez más la presencia de hombres y mujeres capaces de actuar con criterio en la búsqueda de soluciones a los conflictos, cualquiera que sea su campo de acción.

Quienes cuentan con una formación profesional tienen una responsabilidad mayor en este proceso, dado que la distancia entre países ricos y pobres hace que para una gran mayoría el ser profesional sea un privilegio de pocos. Por otra parte, en manos de ese reducido grupo se encuentran las decisiones que afectarán a grupos humanos amplios.

Plantear la educación en términos de aprendizaje supone tratar al estudiante como un ser más pasivo que activo, subestimando su capacidad para investigar, condición fundamental para el desarrollo de un pensamiento crítico.

La UNESCO ha planteado el desarrollo del pensamiento crítico como uno de los principios fundamentales para la educación superior, la vertiginosidad con la cual en el mundo de hoy se genera nueva información está demandando la puesta en marcha de estrategias para auto-dirigir el propio aprendizaje a lo largo de la vida en los nuevos profesionales.
Las instituciones educativas, particularmente las universidades, reconocen la importancia de promover el aprendizaje auto-dirigido y que este sea continuado a lo largo de la vida; tal reconocimiento las ha llevado a algunas a desarrollar alternativas que han afectado los planes de estudio y las prácticas pedagógicas, y han supuesto la realización de programas especiales para el fortalecimiento de aquellas habilidades de pensamiento y competencias prácticas y cognitivas que cada disciplina considera relevantes para la formación profesional de sus egresados.

La globalización actual en todos los aspectos de la vida, requiere de profesionales capaces, por ello se hace imperativo mejorar el proceso de aprendizaje. Existen países que han avanzado en el desarrollo del pensamiento crítico obteniendo resultados positivos, reflejados en el nivel que demuestran.

La realidad de América Latina en el sistema educativo no es convincente por el desinterés de los gobiernos de turno, al no aplicar políticas acorde a nuestra realidad.

En nuestro país el gran problema son las políticas de gobierno que ensayan con el sistema educativo, lo cual ha dado paso a una educación mediocre.

En un informe del progreso educativo en América latina (2006:6) que fue auspiciado por la UNESCO y la "Organización para la Cooperación y el Desarrollo Económico" (OCDE), afirma que los estudiantes tienen un bajo rendimiento en las evaluaciones de PISA de 2003, los estudiantes de 15 años de edad de Brasil, México, y Uruguay obtuvieron puntajes cercanos al extremo inferior en lectura, matemáticas y ciencias entre los 41 países examinados.

Más de la mitad de los estudiantes latinoamericanos tuvo serias dificultades para utilizar la lectura y ampliar sus conocimientos y habilidades. Según el Instituto de Estadística de la UNESCO (2000), más del 50 por ciento de la población es- 
colar en Perú se ubica en el Nivel 1 o por debajo (índica que los estudiantes tienen serias dificultades para utilizar la lectura como un instrumento para avanzar y ampliar sus conocimientos y destrezas en otras áreas), indicando que, en el mejor de los casos, sólo se pueden manejar las tareas más básicas de la lectura y por ende el poco o casi nada desarrollo del pensamiento crítico.

En consecuencia, el bajo desarrollo del pensamiento crítico en los alumnos de las diferentes instituciones educativas del país tiene mucho que ver con lo poco que leen.

Por ello es de vital importancia que frente a la innovación y proliferación de programas curriculares, se tenga en cuenta en la formación del alumno, el desarrollo del pensamiento crítico tomándolo como eje motor y célula de la educación.

El Ministerio de educación apuesta por una educación que favorezca la capacidad crítica y reflexiva, el dialogo, la concertación, la armonía y es consciente de la importancia de la comunicación como llave para acceder a diversos aprendizajes. Por esta razón continúa impulsando, en el marco del programa de emergencia educativa.

La movilización nacional "Un Perú que lee un país que cambia" que busca la colaboración de todos los actores sociales y políticos en la concreción de aprendizajes fundamentales que se relacionan con las capacidades comunicativas.

Entre los latinoamericanos en el 2001, por ejemplo en lectura Perú ya fue superado por Chile (ahora en el puesto 43), México (46), Brasil (55) y Argentina (57). Esta vez los mismos países están delante del Perú, a los que se agregan Uruguay (49) y Colombia (52) que entraron a PISA desde el 2006. Perú (62) sólo supera ligeramente a Panamá (63), país que recién ingresa a dar estas pruebas. Los mejores puntajes los obtienen los mismos de siempre aunque se van alternando en las posiciones de liderazgo.
Por ejemplo en lectura esta vez puntea Shangai-China que es la única " semi-sorpresa” aunque solo representa a una provincia de toda China- seguido de Corea del Sur, Finlandia, Hong Kong-China, Singapur, Canadá, Nueva Zelanda, Japón, Australia, Holanda, Bélgica, Noruega, Estonia y Suiza.

De lo mucho que hay por aprender de estas pruebas y el análisis de sus resultados, que será motivo de otras columnas, empecemos con una lección clara: los países que estaban en la cabeza en el 2001 lo están en el 2009, y los de la cola del 2001 están en la cola en el 2009, aunque aumentó considerablemente el número y diversidad de países evaluados.

Así mismo, los referentes tradicionales de la educación peruana como Estados Unidos (15 en lectura), Alemania (20), Francia (21), Inglaterra (24) ó España (32), hace tiempo que ya no están entre los líderes del mundo, por lo que bien vale la pena revisar de quiénes nos conviene aprender. Perú subió 43 puntos en lectura entre el 2001 y el 2009, al lado de otros coleros como Chile (40 pts.) Albania (36 pts.) Indonesia (31 pts.) y Letonia (26 pts.). En cambio, subieron menos los punteros como Corea (15 pts.) Hong Kong (8 pts.).

\section{Eso es lógico por dos razones:}

Gracias a todo el esfuerzo de haber salido de la cola aún seguimos entre los coleros, si nos damos saltos grandes en la próxima década. El Perú tendrá un status de un analfabeto mundial.

En la Universidad Nacional de Trujillo, Facultad de Educación, Escuela de Educación Primaria, el bajo nivel del desarrollo del pensamiento crítico se manifiesta en los alumnos ya que no opinan sobre ningún tema de actualidad o sucesos que están marcando la historia de nuestro país, la falta de motivación es generada por el desconocimiento y la improvisación del docente, lo cual acarrea aburrimiento. 
Estas deficiencias sumadas a la realidad socio-económica de Trujillo y de nuestro país, obliga a que la mayoría de nuestros educandos sufran también el impacto de la crisis, pasando a ser colaboradores activos de la manutención del hogar.

Desde esta perspectiva se justifica la investigación orientada a pretender rescatar la importancia y el interés que debe darse a la lectura y al desarrollo del pensamiento crítico, empezando por la orientación de especialistas; aplicando el programa de lectura comprensiva de textos en el curso de comunicación I del I ciclo de la Escuela De Educación Primaria, Facultad De Educación, Universidad Nacional De Trujillo.

\section{Esta situación obliga a preguntarnos:}

¿En qué medida el programa de lectura comprensiva de textos desarrolla el pensamiento crítico de los alumnos del I ciclo de la Escuela Académico Profesional De Educación Primaria, Facultad De Educación, Universidad Nacional De Trujillo, 2010?

\section{Los objetivos que se plantearon fueron:}

1) Determinar el desarrollo del pensamiento crítico a través de un programa de lectura comprensiva de textos en los alumnos del I ciclo de la Escuela Académico Profesional De Educación Primaria, Facultad de Educación, Universidad Nacional De Trujillo, 2010.

2) Evaluar los niveles de la comprensión lectora que ayuden a desarrollar el pensamiento crítico en los alumnos.

3) Estructurar un programa de lectura comprensiva de textos para desarrollar los niveles del pensamiento crítico.

\section{MÉTODOS Y MATERIALES}

\section{Objeto del estudio}

El objeto de estudio estuvo constituido por los alumnos del I ciclo de la Escuela Académico Profesional de Educación Primaria, Facultad De Educación, Universidad Nacional De Trujillo durante el año 2014.

\section{Población}

La población estuvo constituida por 72 alumnos del I ciclo de la Escuela Académico Profesional de Educación Primaria, Facultad de Educación, Universidad Nacional de Trujillo

\section{Muestra}

La muestra estuvo constituida por dos grupos: Grupo Experimental y Grupo Control, cada uno formado por 30 hombres y 42 mujeres, en total 72 alumnos del I ciclo de la Escuela Académico Profesional de Educación Primaria, Facultad de Educación, Universidad Nacional de Trujillo, a quienes se aplicó el diseño de pre test y post test.

\section{Instrumentos de recolección de datos}

-Ficha de observación. - Permite recoger información sobre determinadas características personales; al igual que la lista de cotejos, se utilizaron en la observación en las sesiones programadas.

-Fichas textuales. - Para citar algunos párrafos importantes de la nuestra investigación

-Fichas de resumen. - Para organizar sintéticamente las ideas principales encontradas en una o más páginas de una determinada lectura.

-Encuestas. - Para obtener información de los alumnos acerca de la práctica de la lectura y de la utilización de organizadores visuales.

-Cuestionario pre-test y post-test. - Servirán para establecer comparaciones de los puntajes obtenidos por el grupo experimental y el grupo control.

\section{Métodos y Técnicas}

-Método cuantitativo, cuya recolección de datos fue a través de un test, la misma que se empleará antes y después de la experiencia.

-Método explicativo, deseamos investigar acerca de las causas del porque nuestro estudiante uni- 
versitario no critica en los diversos espacios sociales del mundo que lo rodea.

\section{Técnica}

-Observación

\section{Diseño de estudio}

La investigación fue cuasi-experimental, aplicándose el diseño de pre-test y post-test con dos grupos, el grupo experimental y el grupo control. Posteriormente, ambos grupos fueron evaluados.

\section{Procedimiento:}

-Se coordina con los alumnos para desarrollar y aplicar el Programa con los estudiantes del I ciclo de Educación

-Se elaboró el pre test, para conocer la comprensión lectora de los alumnos de la muestra.

-Luego se aplicó el programa de comprensión lectora a los alumnos del I ciclo de Educación
-Después se aplicó el post test.

-Posteriormente los datos se procesaron, analizaron y se discutieron

-Se redactó las conclusiones y sugerencias en base a la discusión de resultados

-Se comunicaron las conclusiones del trabajo de investigación.

\section{Calificación del pre-test y post-test}

Escala nominal

Escala ordinal

Excelente

17-20

Bueno

13-16

Regular

$09-12$

Deficiente

05-08

Malo

00-04

\section{RESULTADOS}

Resultados del test de comprensión lectora aplicado a los del I ciclo de la Escuela Académico Profesional de Educación Primaria, Facultad de Educación, Universidad Nacional de Trujillo.

Tabla $\mathrm{N}^{\circ} 1$. Nivel literal de la comprensión lectora de los alumnos del I ciclo de la Escuela Académico Profesional de Educación Primaria, Facultad de Educación, Universidad Nacional de Trujillo, grupo experimental y control: pre-test y post-test

\begin{tabular}{|c|c|c|c|c|c|c|c|c|}
\hline \multirow{4}{*}{$\begin{array}{l}\text { Nivel literal de } \\
\text { comprensión } \\
\text { lectora }\end{array}$} & \multicolumn{8}{|c|}{ Grupos } \\
\hline & \multicolumn{4}{|c|}{ Experimental } & \multicolumn{4}{|c|}{ Control } \\
\hline & \multicolumn{2}{|c|}{ Pre Test } & \multicolumn{2}{|c|}{ Postest } & \multicolumn{2}{|c|}{ Pre Test } & \multicolumn{2}{|c|}{ Postest } \\
\hline & Alumnos & $\%$ & Alumnos & $\%$ & Alumnos & $\%$ & Alumnos & $\%$ \\
\hline Excelente & 4 & 5,6 & 22 & 31 & 3 & 4 & 15 & 21 \\
\hline Bueno & 12 & 16,7 & 36 & 50 & 10 & 14 & 28 & 39 \\
\hline Regular & 20 & 27,8 & 14 & 19 & 21 & 29 & 29 & 40 \\
\hline Deficiente & 21 & 29,1 & 0 & 0 & 22 & 31 & 0 & 0 \\
\hline Malo & 15 & 20,8 & 0 & 0 & 16 & 22 & 0 & 0 \\
\hline Total & 72 & 100 & 72 & 100 & 72 & 100 & 72 & 100 \\
\hline
\end{tabular}


Figura No 1 . Nivel literal de la comprensión lectora de los alumnos del I ciclo de la Escuela Académico Profesional de Educación Primaria, Facultad de Educación, Universidad Nacional de Trujillo, grupo experimental y control: pre-test y post-test

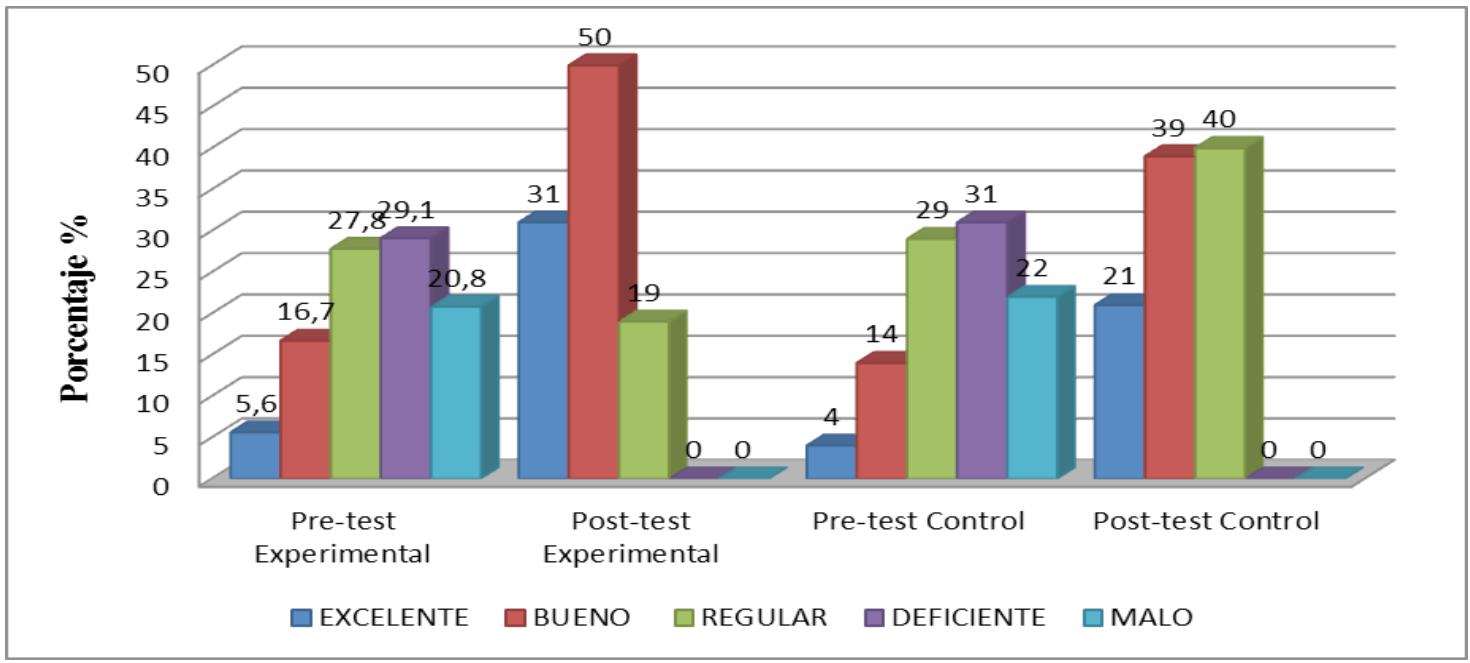

En la Tabla y Fig. N$^{\circ} 1$ muestra en el "nivel literal" de la comprensión lectora según el pre y post test del grupo experimental y control respectivamente el cual indica que, en el grupo experimental en el pre - test el 5,6\% (4) alumnos están en la escala de los excelentes, un $27,8 \%$ (20) alumnos están regulares, un 20,8\% (15) alumnos en la escala de malo; y en el post test del mismo grupo el 50\% (36) alumnos están en la escala de bueno, el 31\% (22) están la escala de los excelentes, el 19\% (14) en los regulares y hay $0 \%$, es decir ningún alumno representa esta escala de deficientes y malos. Respecto al grupo control en el pre test 31\% (22) representan a la escala de deficientes, 29\% (21), se encuentran en la escala de regular, y el $22 \%$ (16) pertenece a la escala malos; dentro de este grupo en el post test hay un 40\% (29) dentro de la escala regular, 39\% (28) están en la escala de buenos, $21 \%$ (15) en la escala de los excelentes y los deficientes y malos representan el $0 \%$

Tabla № 2. Nivel inferencial de la comprensión lectora de los alumnos del I ciclo de la Escuela Académico Profesional de Educación Primaria, Facultad de Educación, Universidad Nacional de Trujillo, grupo experimental y control: pre-test y post-test

\begin{tabular}{|c|c|c|c|c|c|c|c|c|}
\hline \multirow{4}{*}{$\begin{array}{l}\text { Nivel inferencial de } \\
\text { la comprensión } \\
\text { lectora }\end{array}$} & \multicolumn{8}{|c|}{ Grupos } \\
\hline & \multicolumn{4}{|c|}{ Experimental } & \multicolumn{4}{|c|}{ Control } \\
\hline & \multicolumn{2}{|c|}{ Pre Test } & \multicolumn{2}{|c|}{ Postest } & \multicolumn{2}{|c|}{ Pre Test } & \multicolumn{2}{|c|}{ Postest } \\
\hline & Alumnos & $\%$ & Alumnos & $\%$ & Alumnos & $\%$ & Alumnos & $\%$ \\
\hline Excelente & 4 & 6 & 17 & 24 & 4 & 6 & 17 & 24 \\
\hline Bueno & 7 & 10 & 16 & 22 & 8 & 11 & 23 & 32 \\
\hline Regular & 24 & 33 & 37 & 51 & 27 & 38 & 29 & 40 \\
\hline Deficiente & 19 & 26 & 2 & 3 & 17 & 24 & 3 & 4 \\
\hline Malo & 18 & 25 & 0 & 0 & 16 & 22 & 0 & 0 \\
\hline Total & 72 & 100 & 72 & 100 & 72 & 100 & 72 & 100 \\
\hline
\end{tabular}


Figura $\mathrm{N}^{\circ}$ 2. Nivel inferencial de la comprensión lectora de los alumnos del I ciclo de la Escuela Académico Profesional de Educación Primaria, Facultad de Educación, Universidad Nacional de Trujillo, grupo experimental y control: pre-test y post-test

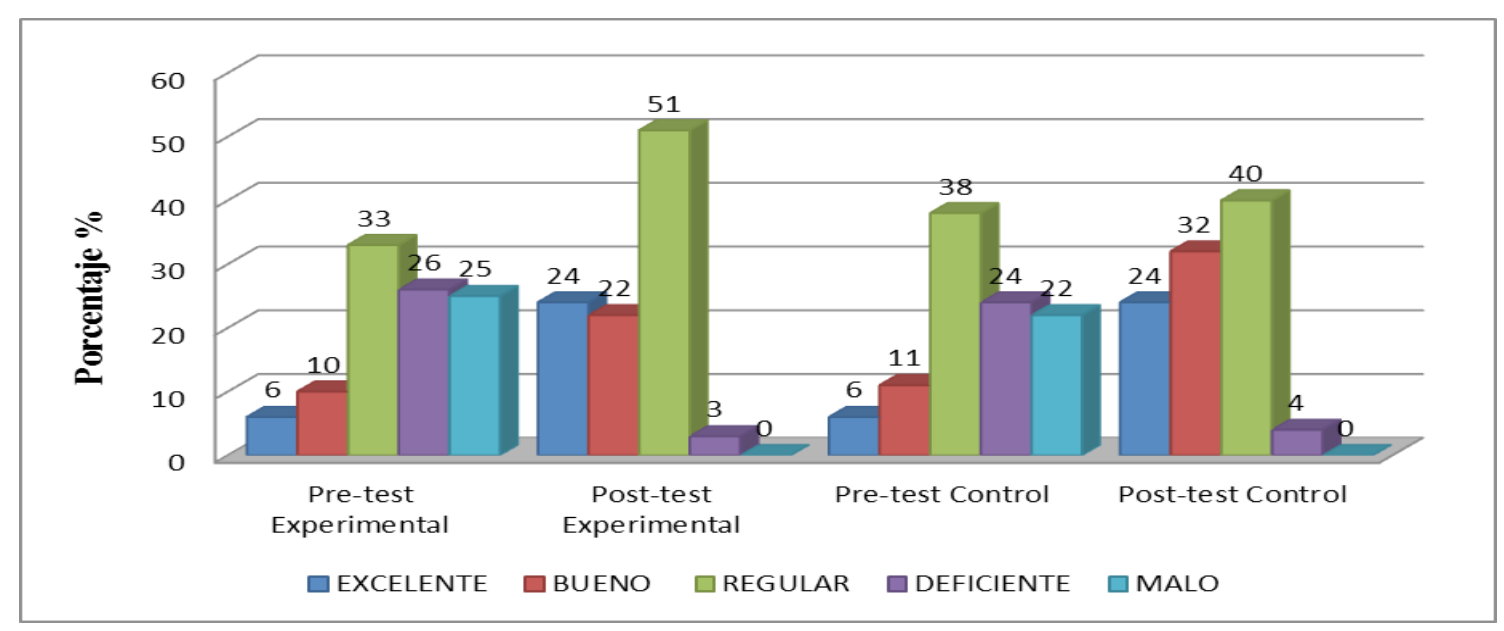

En la tabla y fig. $\mathrm{N}^{\circ} 2$ muestra en el "nivel inferencial" de la comprensión lectora según el pre $\mathrm{y}$ post test del grupo experimental y control respectivamente el cual indica que, en el grupo experimental en el pre-test el 33\% (24) alumnos están en la escala de regular, el 26\% (19) y el 25\% (18) se encuentran en la escala de deficiente y malo respectivamente; y en el post test del mismo grupo el 51\% (37) alumnos están en la escala de regular, el 24\% (17) están la escala de los excelentes, el 22\% (16) en los buenos, hay un
$0 \%$, para la escala de malos y solo un $3 \%$ (2) en la escala de deficientes. Respecto al grupo control en el pre test 38\% (27) representan a la escala de regular, 24\% (17), se encuentran en la escala de deficientes, el 22\% (16) pertenece a la escala malos; dentro de este grupo en el post test hay un $40 \%$ (29) alumnos que se encuentran dentro de la escala regular, $32 \%$ (23) están en la escala de buenos, el 24\% (17) pertenecen a la escala de los excelentes y solo un $4 \%$ (3) pertenecen a la escala de deficientes y malos representan el $0 \%$

Tabla No 3. Nivel crítico de la comprensión lectora de los alumnos del I ciclo de la Escuela Académico Profesional de Educación Primaria, Facultad de Educación, Universidad Nacional de Trujillo, grupo experimental y control: pre-test y post-test

\begin{tabular}{|c|c|c|c|c|c|c|c|c|}
\hline \multirow{4}{*}{$\begin{array}{l}\text { Nivel inferencial de } \\
\text { la comprensión } \\
\text { lectora }\end{array}$} & \multicolumn{8}{|c|}{ Grupos } \\
\hline & \multicolumn{4}{|c|}{ Experimental } & \multicolumn{4}{|c|}{ Control } \\
\hline & \multicolumn{2}{|c|}{ Pre Test } & \multicolumn{2}{|c|}{ Postest } & \multicolumn{2}{|c|}{ Pre Test } & \multicolumn{2}{|c|}{ Postest } \\
\hline & Alumnos & $\%$ & Alumnos & $\%$ & Alumnos & $\%$ & Alumnos & $\%$ \\
\hline Excelente & 0 & 0 & 18 & 25,0 & 1 & 1 & 15 & 21 \\
\hline Bueno & 4 & 6 & 14 & 19,4 & 4 & 6 & 27 & 38 \\
\hline Regular & 36 & 50 & 37 & 51,4 & 28 & 39 & 29 & 40 \\
\hline Deficiente & 13 & 18 & 2 & 2,8 & 21 & 29 & 1 & 1 \\
\hline Malo & 19 & 26 & 1 & 1,4 & 18 & 25 & 0 & 0 \\
\hline Total & 72 & 100 & 72 & 100 & 72 & 100 & 72 & 100 \\
\hline
\end{tabular}

Fuente: Base de datos Excel del test de comprensión lecto 
Figura No 3. Nivel crítico de la comprensión lectora de los alumnos del I ciclo de la Escuela Académico Profesional de Educación Primaria, Facultad de Educación, Universidad Nacional de Trujillo, grupo experimental y control: pre-test y post-test

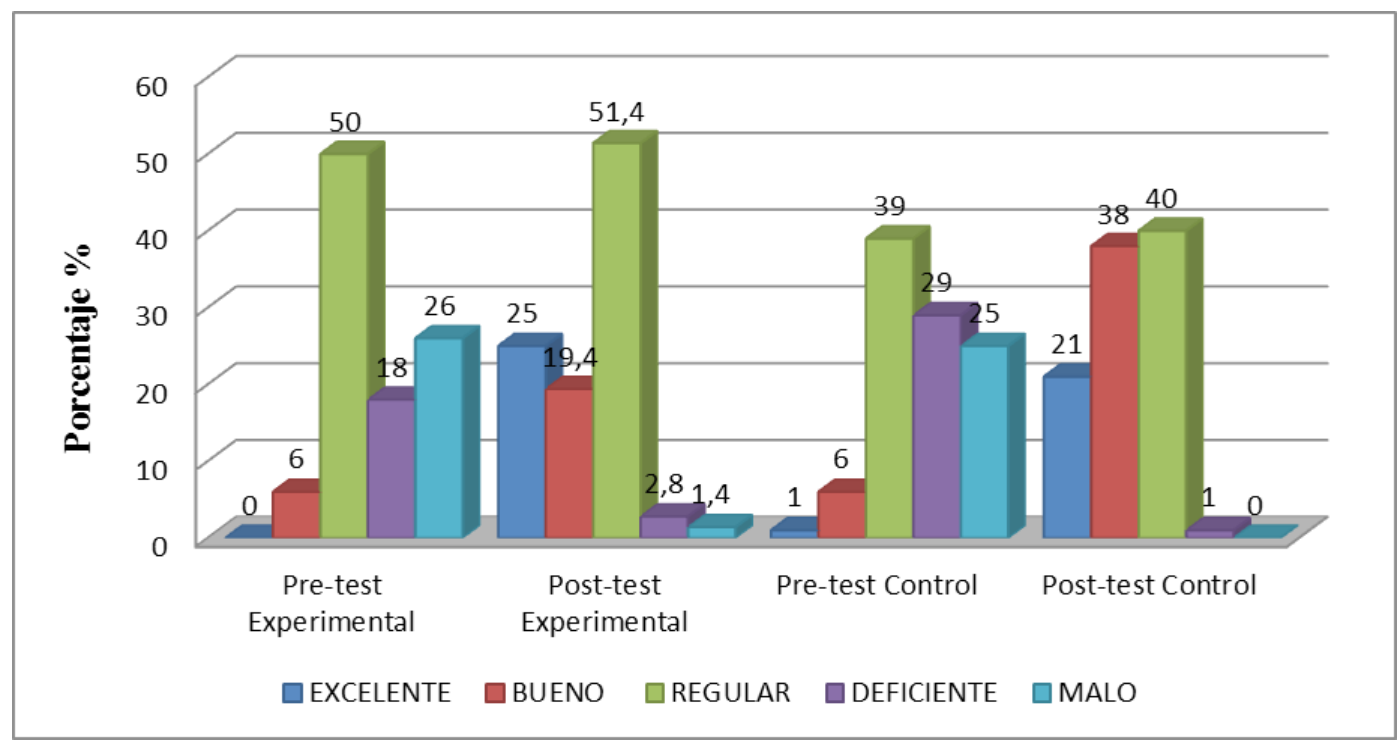

En la tabla y figura $\mathrm{N}^{\circ} 3$ muestra en el "nivel crítico" de la comprensión lectora según el pre y post test del grupo experimental y control respectivamente el cual indica que, en el grupo experimental en el pre-test el 50\% (36) alumnos están en la escala de regular, el $26 \%$ (19) y el $18 \%$ (13) se encuentran en la escala de deficiente y malo respectivamente; $y$ en el post test del mismo grupo el 51,4\% (37) alumnos están en la escala de regular, el 25\% (18) están la escala de los excelentes, el 19,4\% (14) en la escala bueno y el 2,8\% (2) y
$1,4 \%$ (1) alumnos se encuentran en la escala de deficientes y malos. Respecto al grupo control en el pre test $39 \%$ (28) representan a la escala de regular, 29\% (21), se encuentran en la escala de deficientes, el 25\% (18) pertenece a la escala malos; dentro de este grupo en el post test hay un $40 \%$ (29) alumnos que se encuentran dentro de la escala regular, $38 \%$ (27) están en la escala de buenos, el 21\% (15) pertenecen a la escala de los excelentes y el 1\% (1) pertenecen a la escala de deficientes y malos representan el $0 \%$.

Tabla No 4. Estadística descriptiva de los puntajes de los niveles de la comprensión lectora de los alumnos del I ciclo de la Escuela Académico Profesional de Educación Primaria, Facultad de Educación, Universidad Nacional de Trujillo, grupo experimental y control: pre-test y post-test

\begin{tabular}{|c|c|c|c|c|c|}
\hline Variable & Gru & & $\begin{array}{c}\text { Valor } \\
\text { Mínimo }\end{array}$ & $\begin{array}{c}\text { Valor } \\
\text { Máximo }\end{array}$ & Media \\
\hline \multirow{4}{*}{ Nivel } & \multirow{2}{*}{ Experimental } & Pre Test & 2 & 20 & 2.84 \\
\hline & & Post Test & 9 & 20 & 2.41 \\
\hline & \multirow{2}{*}{ Control } & Pre Test & 6 & 20 & 1.75 \\
\hline & & Post Test & 11 & 20 & 1.17 \\
\hline \multirow{4}{*}{ Nivel } & \multirow{2}{*}{ Experimental } & Pre Test & 2 & 19 & 1.44 \\
\hline & & Post Test & 6 & 20 & 1.3 \\
\hline & \multirow{2}{*}{ Control } & Pre Test & 1 & 19 & 1.4 \\
\hline & & Post Test & 7 & 20 & 1.05 \\
\hline \multirow{4}{*}{ Nivel } & \multirow{2}{*}{ Experimental } & Pre Test & 2 & 16 & 1 \\
\hline & & Post Test & 3 & 19 & 1.44 \\
\hline & \multirow{2}{*}{ Control } & Pre Test & 3 & 18 & 1.23 \\
\hline & & Post Test & 7 & 19 & 1.27 \\
\hline
\end{tabular}


De la tabla No 4 , se observa las estadisticas descriptivas, puntaje del nivel literal del grupo experimental; pre-test se encontró un valor mínimo de 02 puntos y un valor máximo de 20 puntos, una media aritmética de 2.84; en el grupo posttest se encontró un valor mínimo de 09 puntos y un valor máximo de 20 puntos, una media aritmética de 2.41. En el grupo de control; el pre-test, encontró un valor mínimo de 06 puntos, un valor máximo de 20, una media de 1.75; en el grupo post-test se encontró un valor mínimo de 11, un valor máximo de 20 puntos y una media de 1.17. En el puntaje del nivel inferencial del grupo experimental; pre-test se encontró un valor mínimo de 02 puntos, un valor máximo de 19 , y una media de 1.44; en el grupo post-test, se encontró un valor mínimo de 06 , un valor máximo de 20 y una media de 1.30. En el grupo control, el pretest se encontró un valor mínimo de 01 , un valor máximo de 19 y una media de 1.40; en el grupo post-test, se encontró un valor mínimo de 07 , un valor máximo de 20 puntos y una media de 1.05 . En el puntaje de nivel crítico del grupo experimental; pre-test se encontró un valor mínimo de 02 , un valor máximo de 16 y una media de 1 ; en el grupo post-test se encontró un valor mínimo de 03, un valor máximo de 19 y una media de 1.44; en el grupo control, el pre-test se encontró un valor mínimo de 03 , un valor máximo de 18 y una media de 1.23; en el grupo post-test, se encontró un valor mínimo de 07 , un valor máximo de 19 y una media de 1.27

\section{Prueba de homogeneidad}

La prueba de homogeneidad s es un estudio comparativo de la respuesta observada en los dos grupos por lo cual se plantea las siguientes hipótesis.

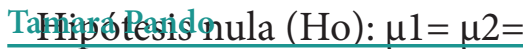

En la cual nos permite afirmar que las medias de los grupos son iguales y los grupos proceden de poblaciones con medias iguales.

-Hipótesis alternativa (H1): al menos uno de los grupos tiene una media distinta

Tabla No 5. Prueba de homogeneidad de varianzas de la comprensión lectora

\begin{tabular}{ccccc} 
& $\begin{array}{c}\text { Estadístico de } \\
\text { Levene }\end{array}$ & gl1 & gl2 & Sig. \\
\hline Pre-test &, 127 & 1 & 54 &, 723
\end{tabular}

\begin{tabular}{|c|c|c|c|c|c|c|}
\hline & & $\begin{array}{l}\text { Suma de } \\
\text { cuadrados }\end{array}$ & gl & $\begin{array}{c}\text { Media } \\
\text { cuadrática }\end{array}$ & $\mathbf{F}$ & Sig. \\
\hline \multirow{3}{*}{ Pre-test } & Inter-grupos & 1,143 & 1 & 1,143 &, 005 & ,945 \\
\hline & Intra-grupos & $12,984,571$ & 54 & 240,455 & & \\
\hline & Total & $12,985,714$ & 55 & & & \\
\hline \multirow{3}{*}{ Pos-test } & Inter-grupos & $16,354,446$ & 1 & $16,354,446$ & 79,817 &, 000 \\
\hline & Intra-grupos & $11,064,536$ & 54 & 204,899 & & \\
\hline & Total & $27,418,982$ & 55 & & & \\
\hline
\end{tabular}


Siendo el valor de $\mathrm{p}=, 945$ en el pretest por lo cual se afirma que son grupos homogéneos mientras que en el postes $\mathrm{p}=, 000$ por lo cual se rechaza la hipótesis nula aceptando la hipótesis alterna que existe una diferencia de medias en el postes del grupo control con el grupo experimental debido a que el programa de lectura comprensiva de textos desarrollo significativamente el pensamiento crítico en los alumnos de la universidad Nacional de Trujillo.

\section{DISCUSIÓN}

Los resultados de esta investigación son relevantes debido a que su sistematización ha permitido demostrar que el programa de lectura comprensiva de textos desarrolla significativamente el pensamiento crítico en los estudiantes de la muestra de los grupos experimental y control.

Iniciaremos citando a Cáceda, F. (2002) quien estudió la capacidad para la lectura en el nivel secundario en Trujillo. Llegando a las siguientes conclusiones: la lectura es la base importante del enriquecimiento cultural; el niño y adolescente peruano casi no lee; las restricciones económicas imposibilitan la presencia de libros y revistas en Pensamiento crítico, en los al limnos de la Universida

En efecto, la lectura supone la práctica más importante para el estudio, nos ayuda a entender y recordar; se desarrolla el pensamiento crítico, cuando al leer un texto, se saca las conclusiones y se contrasta con lo que se piensa; esta acción ayuda a entender el texto y recordarlo.

En ese sentido, tal como se observa en la Fig. $\mathrm{N}^{\circ}$ 1, en el nivel literal de la comprensión lectora de los alumnos del I ciclo de la Escuela de Educación Primaria, Facultad de Educación, de la Universidad Nacional de Trujillo, en los porcentajes obtenidos en el pre-test, de los grupos experimental y control, ambos mostraron un gran porcentaje de deficiencia y mala comprensión de lectura; sin embargo luego de aplicar el post-test se observa que el porcentaje aumenta significativamente; es decir, antes de aplicar el programa hubieron varias dificultades lecturales: lento ritmo de lectura en más de la mitad de estudiantes y entendimiento difícil del vocabulario contenido en los textos leídos por la mayoría de estudiantes.
Esto se debe a que, los alumnos, al leer no comprendían cabalmente los textos que requerían consultar en las diversas asignaturas de su especialidad, por lo que, para desarrollar sus tareas académicas, estudiar para sus exámenes y aprender los contenidos de tal o cual asignatura, no lo harían priorizando libros sino preferían sus tradicionales copias o apuntes realizados en clases, con las implicancias cognitivas y académicas que este hecho traería.

De la misma manera, se observa en el nivel inferencial (Fig. No 2), en el pre-test del grupo experimental y control, sus porcentajes de deficiencia y mala comprensión de lectura son muy elevados; al aplicar el post-test, de igual manera como

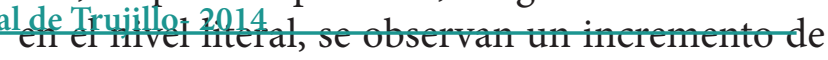
porcentaje considerable. Esos bajos niveles de comprensión lectora en los estudiantes, se debe a que la mayoría de ellos tuvieron docentes en Educación Secundaria que no les enseñaron en forma metódica o sistemática a leer comprensivamente durante el desarrollo de sus clases, teniendo en cuenta que la lectura comprensiva se debe enseñar antes del ingreso a la Universidad.

En efecto, González Moreyra (1998) revela en su investigación que muchos estudiantes universitarios iniciales de Lima, tienen deficiencias lectoras especialmente en los textos vinculados a las ciencias, las humanidades y la literatura, es decir, tienen problemas de comprensión lectora, y por ende, no comprenden bien lo que leen. Deficiencias que son comunes o análogas a las observadas en los estudiantes de nuestra muestra, que expresan su dificultad en entender el contenido de los textos que leen.

En el nivel crítico (Fig. № 3), en el pre-test en el grupo experimental y en el grupo control, los porcentajes son significativos en la escala regular 
y luego del post-test esos porcentajes se incrementaron y se distribuyeron en las escalas regular, bueno y excelente.

A nivel internacional, según Carnero y Salinas (1999) la mayoría de estudiantes universitarios iniciales de una Facultad Pedagógica española presentan relevantes dificultades de comprensión lectora, referidas principalmente a la no utilización de estrategias metacognitivas y al uso de viejas fórmulas de resolución de actividades de estudio. Dificultades, que igualmente se observan en los estudiantes de la muestra, y se reflejan en sus bajas puntuaciones en la prueba del pretest.

Una preocupación seria de los investigadores universitarios, constituye las dificultades y problemas en la comprensión lectora de sus alumnos, quienes vienen proponiendo el desarrollo de múltiples conceptos, hipótesis, teorías, enfoques, metodologías de investigación, relacionadas con el estudio de la comprensión lectora aplicando un pensamiento crítico como enseñanza universitaria óptima para mejorar la comprensión lectora en los estudiantes.

Cabe agregar, con respecto a la comparación de medias para muestras independientes (control experimental) y relacionados (pre - post test) de los puntajes de aprendizaje significativo por niveles: Nivel literal, nivel inferencial y nivel crítico se muestran con capacidad de diferenciar entre niveles, comprender lo que leen, más seguros y confiados al emitir sus juicios y de manera general en el nivel de comprensión de información.

Concluimos con Becerra P. (2000) quien señala que la aplicación de un programa de lectura a los alumnos, mejora el aprendizaje de la entonación de la palabra en forma significativa. Además, leer en voz alta con entonación e interpretación adecuada es una indicación de la creciente capacidad del alumno para retener la información, esto corresponde al nivel inferencial del pensamiento. Esto se comprueba en la investigación ya que se demostró que la aplicación de un programa de lectura comprensiva de textos en los alumnos universitarios desarrolla significativamente el pensamiento crítico en todos sus niveles.

\section{CONCLUSIONES}

a) La aplicación del programa de lectura comprensiva de textos desarrolla significativamente los niveles del pensamiento crítico en los alumnos del I ciclo de la Escuela Académico Profesional De Educación Primaria, Facultad De Educación, Universidad Nacional De Trujillo, 2014.

b) La aplicación del programa de lectura comprensiva de textos desarrolla significativamente el pensamiento crítico en todos sus niveles.

c) La lectura es la base importante para el aprendizaje y el enriquecimiento cultural de los alumnos universitarios.

d) La aplicación del programa de lectura comprensiva en el aspecto universitario, promueve el desarrollo del pensamiento crítico en los estudiantes, con un enfoque positivo.

e) La actividad de comprensión de lectura debe ser reforzada desde el nivel educativo, de tal manera, que la lectura contribuya a la formación de un pensamiento crítico en los alumnos universitarios, con la finalidad de lograr competencias laborales y asegurar la empleabilidad de los estudiantes, contribuyendo así al desarrollo local, regional y nacional.

\section{REFERENCIAS BIBLIOGRÁFICAS}

Aduna A. (1987) Curso de Hábitos de estudio y autocontrol. Editorial Trillas. México; 91.

Alliende F. (1990) Prueba CLP Formas paralelas. Ediciones Universidad Católica de Chile. Chile; 52.

Alliende F. (1991) Comprensión de la Lectura. Editorial Andrés Bello. Chile; 45.

Alliende F. Condemarín M. (1986) La lectura: teoría, evaluación y desarrollo. 2º edición. Editorial Andrés Bello. Chile; 264. 
Argudin Y. Luna M. (2001). Libro del profesor (desarrollo del pensamiento crítico). Editorial Plaza y Valdez. México; 220

Becerra P. (2000). Influencia de un programa de lectura en el aprendizaje de la entonación de la palabra dirigida a 40 alumnos del cuarto grado de educación primaria del C.E. $N^{\circ}$ 80392 de Chepen.

Botkin R. (1980). Pensamiento un estudio experimental y social. Editorial Oikus-Tau. España; 24.

Cáceda F. (2002). Como acrecentar la capacidad para la lectura en el nivel secundario del primer año de educación secundaria del colegio "Sánchez Carrión" de Trujillo. Universidad Nacional de Trujillo. Perú.

De Vega M. (1994). Introducción a la Psicología Cognitiva. Editorial Alianza. España; 65.

Dewey J. (1967). La concepción democrática en educación, el pensamiento y conocimiento. Editorial Urano. España; 32.

De Zubiría J. (1995). Orígenes del pensamiento. Fundación Alberto Merani. Colombia; 253.

Eggen P. y Kauchak D. (1999). Estrategias Docentes: Enseñanza de Contenidos Curriculares y Desarrollo de Métodos de Pensamiento. Editorial FCE. Argentina; 388.

Enríquez L. (1997). Hábitos de lectura y rendimiento académico en la asignatura de Lenguaje y Literatura en los alumnos del primer grado de educación secundaria en el Colegio Nacional Mixto No 80017 "Alfredo Tello Salavarría"- Trujillo.

González Moreyra, Raúl (1998). Comprensión lectora en estudiantes universitarios iniciales. Persona, $\mathrm{N}^{\circ} 1$, Lima, pp. 43-65

Klausmeir H. (1997) Psicología educativa. Editorial Urano. España; 67.

Lozano S. (1992). Los senderos del lenguaje. Impreso en los talleres gráficos de Editorial Libertad E.I.R.L. Perú-La Libertad.

Ministerio De Educación. (2005). Indicadores de la educación. Unidad de Estadística Educativa. Perú-Lima.

Ministerio De Educación. (2006). Guía Para el Desarrollo del Pensamiento Crítico. Impreso por Fimart S.A.C. Perú-Lima.

Neisser O. (1983). La constitución social de la inteligencia. Editorial La Merced. España; 55.
Narvate, M. (2007). Lectoescritura. Lima: La tierra.

Navarro López, H. (2008). Los seis Niveles de Lectura, Leer para comprender, comprender para escribir. Quito: Instituto Pedagógico Alfredo Pérez Guerrero.

Piaget, J. (1972). De la lógica del niño a la lógica del adolescente. Editorial Buenos Aires. Argentina; 78-79.

Pinzás J. (1995). Leer pensando. Asociación de investigación aplicada y extensión pedagógico. Perú-Lima; 92.

Quintana H. (2000). La enseñanza de la compresión lectora. Editorial Magisterio. Colombia; 344.

Quintanilla F. (1999). El mejor método para Estudiar. Edit Universo S.A.S.F. Perú-Lima.

Riatiga R, Acosta M, Calabria Y, Paba Z, Prado A (2009). La lectura como generadora del pensamiento crítico. Universidad del Magdalena Vicerrectoría de Investigación Santa Marta. España.

Sánchez H, Reyes C. (1987). Metodología y diseño en la investigación científica. Editorial Manual Moderno. Perú-Li$\mathrm{ma} ; 62$.

Solé I. (2000). Estrategias de lectura. 11º edición. Editorial Grao. España; 178.

Solé I. (2001). Estrategias de Comprensión lectora. Editorial Grao. España; 28 


\section{ANEXO A \\ CUESTIONARIO PARA APLICAR A LOS ESTUDIANTES}

INSTRUCIONES: Te solicitamos leer cuidadosamente cada una de las preguntas siguientes y contestar verazmente marcando con un ASPA (X) en los cuadritos correspondientes a la respuesta escogida. Te agradezco tu colaboración

1. ¿Tus docentes de educación secundaria te han enseñado en forma metódica a leer comprensivamente?
a) $\mathrm{Si}($ )
b) No ( )

2. ¿Recibes una metódica enseñanza de parte de los docentes de tu Facultad sobre comprensión lectora?
a) Frecuentemente ( )
b) a veces ( )
c) nunca

3. ¿Cuando lees, lo haces con un ritmo muy lento?
a) $\mathrm{Si} \mathrm{(} \mathrm{)}$
b) No ( )

¿Porqué?:

4. Cuando lees libros o separatas de tus asignaturas, ¿memorizas lo que lees empleando las mismas palabras del autor?
a) $\mathrm{Si}()$
b) No ( ) ¿Porqué?:

5. ¿Cuando lees libros o separatas de tus asignaturas comprendes fácilmente lo que lees?
a) Frecuentemente
b) A veces
( )
c) nunca
( ) ¿Porqué?:

6. El léxico o vocabulario de los libros o separatas que lees lo encuentras:
a) Demasiado difícil, desconocido y abstracto
b) Medianamente difícil
c) Poco difícil
d) Fácil, conocido y sencillo 


\section{ANEXO B}

\section{LECTURA COMPRENSIVA}

INSTRUCCIONES: Luego de leer atentamente el texto le solicitamos responder sincera y reflexivamente las diversas preguntas de cada texto

\section{LA TAXONOMÍA DE BARRET}

Fuente: http://mundomagico.obolog.com/taxonomia-barret-75570

Los alumnos presentan, aparentemente, muchas limitaciones para aprender a expresar ideas con sentido crítico. Es frecuente -en el aula- que cuando se les pide que expresen su punto de vista sobre un tema determinado muestren muchas limitaciones, sin embargo, en otros temas que están vinculados con su entorno juvenil extracurricular, si son capaces de presentar, con algunas limitaciones, un pensamiento crítico y reflexivo. Este hecho es una muestra de que por un lado presentan esta capacidad como una función mental inferior o espontánea (Vigotsky), entonces, podemos, los profesores, desarrollar en ellos esta capacidad en base a la interacción social que es la enseñanza y podemos llegar a que desarrollen sus funciones mentales superiores.

Si como profesor quiero promover el desarrollo de esta capacidad, entonces, yo debo tener muy claro mi objetivo y el cómo hacerlo. Posiblemente existen muchas formas miles-, pero hay que tratar de buscar una información adecuada y adaptarla a la realidad del estudiante

Una estrategia metodológica con la cual estoy trabajando desde el curso de Comunicación es LA TAXONOMÍA DE BARRET que consiste en la evaluación de textos teniendo en cuenta la elaboración "específica de preguntas". Por ejemplo, después de haber leído un cuento se elabora preguntas de tipo: Literales, Inferenciales, de Organización y Clasificación, Apreciación, y por último preguntas de Evaluación Crítica. Estas últimas buscan que el alumno se exprese libremente (no hay una respuesta exacta) y fundamenten el porqué de su respuesta.

Ahora, en honor a la verdad, más nos preocupamos por hacer preguntas de tipo literales y más aún, solo sabemos hacer preguntas abiertas o múltiples, cuando existen otros tipos como preguntas tipo Clozé, de relación, birrespuestas, y que permiten que el alumno pueda expresar mejor sus pensamientos y pueda ser mejor evaluado.

Una vez más se pone de manifiesto la importancia del enfoque sociocultural de Vigostky que considera al ser humano como el resultado de un proceso histórico y social, donde el lenguaje es vital.

Quiero poner un ejemplo de las preguntas según la taxonomía de Barret y para ello se me ocurre esta historia corta:

"Un niño caminaba alegremente por la vereda de la calle, pero cuando observó a lo lejos su reluciente casa su semblante alegre cambió. Sus pasos se hicieron lentos y no supo cómo iba a explicar el cero ocho que había obtenido en la prueba de comprensión lectora. Recordó que en las pruebas anteriores también había salido con bajas notas, aunque en Matemáticas tenía la más alta nota del aula: 20".

\section{PREGUNTAS LITERALES (Se busca recuperar información)}

1. El niño cambió de semblante al

(Obsérvese que estamos usando una pregunta tipo clozé, que solo es para colocar la/s palabras) 
2. Escribe $\mathrm{V}$ de verdadero o $\mathrm{F}$ de falso en las oraciones según el texto:

a) El niño en ningún momento pensó regresar a su casa (..........)

b) Era un niño que estaba mal en todos los cursos (..........)

(En este caso las preguntas tienen dos alternativas $V o F$.)

PREGUNTAS DE REORGANIZACIÓN (El alumno establece relaciones, resume, sintetiza...)

1. Usa tus propias palabras y en dos líneas trata de resumir el texto leído.

(Se plantea a través de una pregunta abierta)

2. Relaciona los elementos de la derecha con los de la izquierda trazando una línea.

Homo Sapiens

Austrolipitecus (Lucy)

Homo Hábilis

Homo Erectus

Orrorín y Ultumay
Desarrolla la habilidad de hacer instrumentos y utensilios.

Grandes primates que por la necesidad de sobrevivir se ponen de vié.

Descubre el fuego y se lanza a la conquista del mundo

Especie mejor adaptada, sobrevive comiendo vegetales cada vez más duros

Atraviesa el mar y vive una verdadera expansión artística, aparecen las pinturas rupestres

(La actividad requiere relacionar las columnas)

PREGUNTAS DE COMPRENSIÓN INFERENCIAL (El alumno debe hipotetizar a partir de lo que se encuentran en el texto)

1. ¿Qué crees que le pasó al niño cuando llegó a casa?

2. ¿Por qué crees que este niño tenía problemas en las pruebas de comprensión?

(Las clásicas preguntas abiertas que abundan en las pruebas de comprensión)

PREGUNTAS DE LECTURA CRÍTICA (Busca que el alumno emita un juicio valorativo, que use sus propios valores)

1. Según la historia que hemos leído ¿tú crees que es correcto que los alumnos siempre destaquen y tengan buenas notas en todos los cursos?

Explícalo según tu punto de vista.

2. Si estuvieses en el mismo caso del alumno, ¿de qué manera enfrentarías este hecho?

(Las preguntas no están regidas por Qué, quién, cómo...; están subordinadas a otra proposición. Genera confianza y más aproximación con el texto)

PREGUNTAS DE APRECIACIÓN (de qué manera el texto genera un impacto psicológico, estético, moral) 
1. ¿Conoces algún caso parecido al que hemos leído? Escríbelo brevemente.

2. ¿Crees que esta historia nos enseña algo importante?; usa tus propias palabras y explícalo.

Finalmente hay que recalcar que la TAXONOMÍA DE BARRET busca formular preguntas específicas que evalúen distintas capacidades como: memoria, secuencia lógica, organización, inferencias, apreciación 